PROCEEDINGS OF THE

AMERICAN MATHEMATICAL SOCIETY

Volume 140, Number 11, November 2012, Pages 3785-3792

S 0002-9939(2012)11242-0

Article electronically published on March 15, 2012

\title{
IRREDUCIBLE CHARACTERS TAKING ROOT OF UNITY VALUES ON $p$-SINGULAR ELEMENTS
}

\author{
GABRIEL NAVARRO AND GEOFFREY R. ROBINSON
}

(Communicated by Jonathan I. Hall)

\begin{abstract}
In this paper we study finite $p$-solvable groups having irreducible complex characters $\chi \in \operatorname{Irr}(G)$ which take root of unity values on the $p$-singular elements of $G$.
\end{abstract}

\section{INTRODUCTION}

If $G$ is a finite group and $p$ is a prime, in this paper we study irreducible complex characters $\chi \in \operatorname{Irr}(G)$ which take root of unity values on the $p$-singular elements of $G$.

There are several reasons to study such characters. In $[\mathrm{NR}$, we gave a proof of a conjecture of J. Carlson, N. Mazza and J. Thévenaz CMT showing that an endo-trivial simple module of a finite $p$-solvable group $G$ with Sylow $p$-subgroups of rank at least 2 is one-dimensional. Recall that, as introduced by E. C. Dade, a simple $K G$-module $V$ over a field $K$ of characteristic $p$ is endo-trivial if

$$
V \otimes V^{*} \cong 1 \oplus P,
$$

where $P$ is a projective module. (Later the second author studied in $\mathrm{R} 2$ lattices satisfying this condition.) Inspired by this idea, we now turn our attention to the set of complex irreducible characters $\operatorname{Irr}(G)$ and consider characters $\chi \in$ $\operatorname{Irr}(G)$ such that

$$
\chi \bar{\chi}=1+\sum_{i} a_{i} \Phi_{\varphi_{i}},
$$

where $\Phi_{\varphi_{i}}$ is the projective indecomposable character of the irreducible Brauer character $\varphi_{i} \in \operatorname{IBr}(G)$. Since $\Phi_{\varphi_{i}}$ vanishes on the $p$-singular elements of $G$, such a character $\chi$ satisfies that $|\chi(x)|=1$ for every $p$-singular $x \in G$, and conversely. (By elementary character theory what we have in fact is that $\chi(x)$ is a root of unity for every $p$-singular $x \in G$. See Lemma 2.2 below.)

Under certain natural circumstances, the cyclic defect theory provides examples of these characters in groups with cyclic Sylow p-subgroups. It turns out that this condition also holds in several simple groups. For instance, the irreducible

Received by the editors December 9, 2010 and, in revised form, December 12, 2010 and May 3, 2011.

2010 Mathematics Subject Classification. Primary 20C15.

The research of the first author is partially supported by the Spanish Ministerio de Educación y Ciencia proyecto MTM2010-15296 and Prometeo/Generalitat Valenciana.

(C)2012 American Mathematical Society Reverts to public domain 28 years from publication 
characters of $P S L\left(2, p^{n}\right)$ of degree $q-1$ take the value -1 on all its $p$-singular elements. Furthermore, if $G$ is a simple group of Lie type of rank 1 in characteristic $r$, then $\mathbf{C}_{G}(x)$ is an $r$-group for each non-trivial $r$-element $x \in G$. Hence, if $\chi$ is the Steinberg character, for each non-trivial $r^{\prime}$-element $y$, we have $\chi(y)= \pm\left|\mathbf{C}_{G}(y)\right|_{r}=$ \pm 1 . In particular, for each prime $p$ other than $r$ which divides $|G|$, we have $|\chi(y)|=$ 1 for each $p$-singular element $y$ of $G$. (As pointed out to us by P. H. Tiep, if $q$ is a power of the prime $p$, then $P S L_{3}(q), P S U_{3}(q), S z(q)$ and ${ }^{2} G_{2}(q)$, among others, also have characters of this type.)

Our interest in this paper is in $p$-solvable groups. In fact, Theorem A below implies our module-theoretic result from $[\mathrm{NR}]$.

Theorem A. Suppose that $G$ is a p-solvable group with Sylow p-subgroups of rank at least 2. Let $\chi \in \operatorname{Irr}(G)$ be faithful taking root of unity values on the p-singular elements of $G$. If $\mathbf{O}_{p}(G)=1$, then $\chi$ is linear.

If the Sylow $p$-subgroups of $G$ are cyclic or generalized quaternion, then there are many examples showing that Theorem $\mathrm{A}$ is false (for instance, in certain Frobenius actions).

Our result in Theorem A shows that under certain circumstances, if the values of an irreducible character on $p$-singular elements are roots of unity, then the same is true everywhere, a fact that can be seen as another example of character values on $p$-singular elements controlling representation-theoretic invariants.

In the case where $\mathbf{O}_{p}(G)>1$, and without assuming $p$-solvability, the situation is also quite tight.

Theorem B. Suppose that $\chi \in \operatorname{Irr}(G)$ is faithful, non-linear, and such that it takes root of unity values on the p-singular elements of $G$. Assume that $P=\mathbf{O}_{p}(G)>1$. Then either $p$ is odd, $P \in \operatorname{Syl}_{p}(G)$ is elementary abelian, $\mathbf{C}_{G}(P)=P \times \mathbf{Z}(G)$, $G / \mathbf{C}_{G}(P)$ acts transitively on $\operatorname{Irr}(P)-\left\{1_{P}\right\}$, and $\chi(1)=|P|-1$, or $p=2$ and $G=S_{4} \times \mathbf{Z}(G)$ with $|\mathbf{Z}(G)|$ odd.

From Theorem B, we see that $G / \mathbf{Z}(G)$ is a doubly transitive permutation group (in the action with $P$ acting by translation on itself, and $G / \mathbf{C}_{G}(P)$ acting by conjugation on $P$ ). Since the finite 2 -transitive groups are known, it is possible to classify all the groups satisfying Theorem B, but we have not attempted this here.

Finally, we mention the work of the second author in R3, in which generalized characters taking root of unity values on all non-identity elements are studied.

\section{Proof}

Let us start with the following.

Lemma 2.1. Suppose that $\xi \in \mathbb{C}$ is a root of unity, and let $e \in \mathbb{Z}$ be an integer. If $e$ divides $\xi$ in the ring of algebraic integers, then $e= \pm 1$.

Proof. We have that $e \alpha=\xi$ for some algebraic integer $\alpha$. Then $1=\xi \bar{\xi}=e^{2} \alpha \bar{\alpha}$, and $\left(1 / e^{2}\right)=\alpha \bar{\alpha}$ is a rational algebraic integer. So $1 / e^{2} \in \mathbb{Z}$.

Our notation for characters mainly follows [I]

Lemma 2.2. Suppose that $\chi \in \operatorname{Irr}(G)$. Then the following conditions are equivalent:

(i) The character $\Psi=\chi \bar{\chi}-1_{G}$ vanishes on the $p$-singular elements of $G$. 
(ii) $|\chi(x)|=1$ for all $p$-singular $x \in G$.

(iii) $\chi(x)$ is a root of unity for all $p$-singular $x \in G$.

Proof. We know that $\Psi=\chi \bar{\chi}-1_{G}$ is a character of $G$ since $1=[\chi, \chi]=\left[\chi \bar{\chi}, 1_{G}\right]$. Now, if $\chi$ is any character of $G, g \in G$ and $|\chi(g)|=1$, it is well known that $\chi(g)$ is a root of unity. (This fact can be found as Problem 3.2 of [I] for instance, and we sketch a proof for the interested reader. Let $K$ be the cyclotomic field of $n$-th roots of unity, of degree $\varphi(n)$ over $\mathbb{Q}$. Let $\alpha \in K$ be an algebraic integer with $|\alpha|=1$. Suppose that $\alpha$ has minimal polynomial $p(x)=\left(x-\alpha_{1}\right)\left(x-\alpha_{2}\right) \cdots\left(x-\alpha_{t}\right) \in \mathbb{Z}[x]$, where the $\alpha_{i}$ 's are the Galois conjugates of $\alpha=\alpha_{1}$ by the group $\operatorname{Gal}(K / \mathbb{Q})$. Hence, $t \leq \varphi(n)$. Now, since $\operatorname{Gal}(K / \mathbb{Q})$ is abelian, Galois conjugation commutes with complex conjugation, and therefore $\left|\alpha_{i}\right|=1$ for all $i$. Now notice that the absolute value of the coefficient of $x^{j}$ in $p(x)$ is at most the binomial coefficient $\left(\begin{array}{l}t \\ j\end{array}\right)$, so there are only finitely many possibilities for $p(x)$. In particular, we deduce that there is only a finite number of algebraic integers $\alpha \in K$ with $|\alpha|=1$. Therefore the set $\left\{\alpha^{m} \mid m \geq 0\right\}$ is finite, and we conclude that $\alpha$ is a root of unity.)

Although we shall not need it in our proofs, we mention now that a character of a finite group vanishes on the $p$-singular elements of $G$ if and only if it is a $\mathbb{Z}$-linear combination of the projective indecomposable characters $\Phi_{\varphi_{i}}$ (by Corollary 2.17 of [N], for instance).

We shall frequently use the following lemma.

Lemma 2.3. Suppose that $\chi \in \operatorname{Irr}(G)$ takes root of unity values on every $p$-singular element $x \in G$. Then the following hold:

(a) If $S \in \operatorname{Syl}_{p}(G)$, then $\chi(1)^{2} \equiv 1 \bmod |S|$. Hence, if $p$ is odd, then $\chi(1) \equiv$ $\pm 1 \bmod |S|$. If $|S|=2^{a}$ and $a \geq 2$, then $\chi(1) \equiv \pm 1 \bmod 2^{a}$ or $\chi(1) \equiv 2^{a-1} \pm$ $1 \bmod 2^{a}$. In any case, $\chi(1)$ is not divisible by $p$.

(b) If $M \triangleleft G$ has order divisible by $p$, then $\chi_{M}$ is a sum of distinct irreducible characters of $M$. In particular, if $\chi$ is non-linear and faithful, then $\mathbf{Z}(G)$ is a $p^{\prime}$-group.

(c) If $M$ is a subgroup of $G$ having a normal p-complement $N$ and a non-trivial Sylow p-subgroup $P$, then

$$
\left[\chi_{M}, \chi_{M}\right]=1+\frac{\left[\chi_{N}, \chi_{N}\right]-1}{|P|} .
$$

Proof. By Lemma 2.2, we can write $\chi \bar{\chi}=1+\Psi$, where $\Psi$ is a character of $G$ such that $\Psi(g)=0$ whenever $g \in G$ is $p$-singular. If $S \in \operatorname{Syl}_{p}(G)$, then we have that $\psi_{S}$ is a multiple of the regular character $\rho_{S}$ of $S$, so $\psi(1)$ is divisible by $|S|$. Then (a) follows. To prove part (b), using Clifford's theorem, write $\chi_{M}=e \sum_{i=1}^{t} \theta_{i}$, where the $\theta_{i} \in \operatorname{Irr}(M)$ are distinct (and $G$-conjugate). If $x \in M$ has order $p$, then

$$
\chi(x)=e\left(\sum_{i=1}^{t} \theta_{i}(x)\right) .
$$

Hence, the root of unity $\chi(x)$ is divisible by $e$ in the ring of algebraic integers, and we apply Lemma 2.1 . 
Suppose finally that $M$ has a normal $p$-complement $N$ and a Sylow $p$-subgroup $P>1$. Then every $g \in M-N$ is $p$-singular. Then

$$
\begin{aligned}
|M|\left[\chi_{M}, \chi_{M}\right] & =\sum_{g \in M}|\chi(g)|^{2} \\
& =\sum_{g \in M-N}|\chi(g)|^{2}+\sum_{g \in N}|\chi(g)|^{2}=(|M|-|N|)+|N|\left[\chi_{N}, \chi_{N}\right],
\end{aligned}
$$

and part (c) easily follows.

We shall also use the following lemma (due to the second author).

Lemma 2.4. Suppose that $\theta$ is a generalized character of an Abelian group A. If $|\theta(a)|=1$ for all $a \in A-\{1\}$, then $\theta=f \rho_{A}-e \mu$, where $\rho_{A}$ is the regular character of $A$, $e$ is a sign, and $\mu \in \operatorname{Irr}(A)$.

Proof. This fact was first stated as a remark after Corollary 4 of [R1]. Another proof is provided in the second paragraph of the proof of Theorem 2.1 of [R3].

Now, we dispose of Theorem B in the introduction.

Theorem 2.5. Suppose that $\chi \in \operatorname{Irr}(G)$ is faithful, non-linear, and such that it takes root of unity values on the p-singular elements of $G$. Assume that $P=$ $\mathbf{O}_{p}(G)>1$. Then either $P \in \operatorname{Syl}_{p}(G)$ is elementary abelian, $\mathbf{C}_{G}(P)=P \times \mathbf{Z}(G)$, $G / \mathbf{C}_{G}(P)$ acts transitively on $\operatorname{Irr}(P)-\left\{1_{P}\right\}$, and $\chi(1)=|P|-1$, or $p=2$ and $G=S_{4} \times \mathbf{Z}(G)$, where $|\mathbf{Z}(G)|$ is odd.

Proof. By Lemma 2.3(a), we know that $\chi$ has degree not divisible by $p$. Hence, the irreducible constituents of $\chi_{P}$ are linear. Since $\chi$ is faithful, we have that $P$ is abelian. Now, by Lemma 2.3(b) and using that $\chi$ is faithful, then we can write $\chi_{P}=\sum_{i=1}^{t} \lambda_{i}$, where $\lambda_{i} \in \operatorname{Irr}(P)$ are distinct and different from $1_{P}$. Thus $\chi(1) \leq|P|-1$. Let $C=\mathbf{C}_{G}(P)$. Since $\left[\chi_{P}, \lambda_{1}\right]=1$ and $P \subseteq \mathbf{Z}(C)$, we easily conclude that $\chi_{C}$ is a sum of distinct linear characters of $C$. Using again that $\chi$ is faithful, we conclude that $C$ is abelian. Hence, we can write $C=P \times N$, where $N=\mathbf{O}_{p^{\prime}}(G)$. Now, write

$$
\chi_{N}=h \sum_{i=1}^{r} \mu_{i},
$$

where $\mu_{i} \in \operatorname{Irr}(N)$ are distinct. Hence $\chi(1)=h r \leq|P|-1$. Also, $\left[\chi_{C}, \chi_{C}\right]=h r$. Now, by Lemma 2.3(c),

$$
h r=1+\frac{h^{2} r-1}{|P|} \leq 1+\frac{h^{2} r-1}{h r+1} .
$$

From this inequality, we conclude that $r=1$ and that $\chi(1)=h=|P|-1$. Hence $\chi_{N}=\chi(1) \mu_{1}$ and since $\chi$ is faithful and $\mu_{1}$ is linear, we get $N \subseteq \mathbf{Z}(G)$. Necessarily $N=\mathbf{Z}(G)$ (since $\mathbf{Z}(G)$ is a $p^{\prime}$-group by Lemma 2.3(b)). Also, since $\chi(1)=|P|-1$, we have that $G / C$ acts faithfully and transitively on $\operatorname{Irr}(P)-\left\{1_{P}\right\}$. In particular, $P$ is an elementary abelian $p$-group.

So we may assume now that $P<S \in \operatorname{Syl}_{p}(G)$. If $p$ is odd, then by Lemma 2.3(a), we have that $\chi(1) \geq|S|-1$, where $S \in \operatorname{Syl}_{p}(G)$. Thus $|P|=|S|$ and $P=S \in$ $\operatorname{Syl}_{p}(G)$, which is not possible. Hence, we have that $p=2$. If $|P|=4$, then $G / C$ is necessarily $S_{3}$ and $G / \mathbf{Z}(G)=S_{4}$. Since $|\mathbf{Z}(G)|$ is odd, by elementary group theory we have that $G=\mathbf{Z}(G) \times S_{4}$. 
Finally, assume that $|P|>4$. By Lemma 2.3(a), we have that $|P|-1=\chi(1) \geq$ $|S| / 2-1$ and therefore $|S|=2|P|$. Since $P$ is elementary abelian and $S$ is not abelian (since $S$ is not contained in $C$ ) it follows that the exponent of $S$ is 4 . Let $\lambda=\operatorname{det}(\chi)$, and let $\lambda_{2}$ be the 2-part of $\lambda$. Now, let $\nu$ be a linear character of $G$ such that $\nu^{\chi(1)}=\bar{\lambda}_{2}$, and notice that $\tau=\nu \chi$ is an irreducible character of $G$ with odd determinantal order which takes root of unity values on the 2 -singular elements of $G$. Since $\operatorname{det}(\tau)$ has odd order, it follows that $\operatorname{det}(\tau)(x)=1$ on 2-elements. Therefore $\tau(x) \equiv \tau(1) \bmod 4$ for every involution $x \in G$. Since $\tau(1)=|P|-1$ and $\tau(x)$ is a sign by hypothesis, we see that $\tau(x)=-1$ for every involution $x \in G$. Now let $U$ be a cyclic subgroup of order 4 . Since $\tau_{U}$ takes root of unity values on the non-identity elements of $U$, it follows by Lemma 2.4 that

$$
\tau_{U}=f \rho_{U}-e \mu
$$

where $e$ is a sign, $\mu \in \operatorname{Irr}(U)$, and $\rho_{U}$ is the regular character of $U$. Now, $|P|-1=$ $4 f-e$ and we deduce that $e=1$ and $4 f=|P|$. Notice that $\operatorname{det}\left(\rho_{U}\right)$ is the unique irreducible character $\lambda$ of $U$ of order 2 . Also, since $\operatorname{det}(\tau)_{U}=1$ and $\tau_{U}=\frac{|P|}{4} \rho_{U}-\mu$, we deduce that $1=\lambda^{\frac{|P|}{4}} \mu^{-1}$. Since 2 divides $|P| / 4$, then we have that $\mu=1$. It follows that $\tau(x)=-1$ for every $x \in G$ of order 4 . Therefore $\tau_{S}=a \rho_{S}-1_{S}$ and $\tau(1) \geq|S|-1$, which is the final contradiction.

We recall that a subgroup $H$ of a finite group $G$ is said to be strongly $p$ embedded in $G$ if $p$ divides $|H|$ and $p$ does not divide $\left|H \cap H^{g}\right|$ for each $g \in G-H$.

The following is an essential part in the proof of Theorem A. We use the Glauberman correspondence, and we refer the reader to [I, Chapter 13] for a reference. But also, we shall use a well-known fact which follows from the Classification of Finite Simple Groups, namely, that the outer automorphism group of a finite simple group of order prime to $p$ has cyclic Sylow $p$-subgroups (see, for example, Theorem 7.1.2 of GLS ).

Theorem 2.6. Suppose that $G$ has a normal p-complement $N$ and that $P \in$ $\operatorname{Syl}_{p}(G)$ is abelian and contains a non-cyclic subgroup of order $p^{2}$. Let $\chi \in \operatorname{Irr}(G)$ such that $\chi(x)$ is a root of unity for every $p$-singular $x \in G$. Then $\chi(1)=1$.

Proof. We argue by induction on $|G|$. By Lemma 2.3(a), we have that $\chi$ has $p^{\prime}$ degree. Hence $\chi_{N}=\mu \in \operatorname{Irr}(N)$ (using Corollary 11.29 of [I] ). Also, if $V$ is an elementary subgroup of $P$ of order $p^{2}$, by considering $N V$ and the character $\chi_{N V} \in \operatorname{Irr}(N V)$, by induction we may assume that $V=P$ is elementary abelian. Now let $\hat{\mu} \in \operatorname{Irr}(G)$ be the canonical extension of $\mu$ to $G$. (This is the unique extension $\tau \in \operatorname{Irr}(N)$ with determinantal order $o(\tau)=o(\mu)$, using Corollary 6.28 of I].) Then, by Corollary 6.17 of $[\underline{I}$, we can write $\chi=\hat{\mu} \lambda$ for some linear $\lambda \in \operatorname{Irr}(P)$, and therefore may assume that $\chi=\hat{\mu}$.

Since $(\hat{\mu})_{N \times \mathbf{O}_{p}(G)}$ is by definition the canonical extension of $\mu$ to $N \times \mathbf{O}_{p}(G)$, we see that $(\hat{\mu})_{N \times \mathbf{O}_{p}(G)}=\mu \times 1_{\mathbf{O}_{p}(G)}$. Hence, if $1 \neq x \in \mathbf{O}_{p}(G)$, then $\hat{\mu}(x)=\mu(1)$ is a root of unity, and we deduce that $\mu(1)=1$. So we may assume that $\mathbf{O}_{p}(G)=1$.

Now we claim that $\hat{\mu}$ takes root of unity values on the $p$-singular elements of $G$ if and only if for every $1 \neq x \in P$, the $\langle x\rangle$-Glauberman correspondent of $\mu$ is linear. If $1 \neq x \in P$ and $y \in \mathbf{C}_{N}(x)$, then we know that

$$
\hat{\mu}(y x)=\epsilon \mu^{*}(y)
$$


where $\mu^{*}$ is the $\langle x\rangle$-Glauberman correspondent of $\mu$ and $\epsilon$ is a sign. (See Theorems 13.6 and 13.14 of $\left[\mathbf{I}\right.$.) If $\mu^{*}$ is linear for every $1 \neq x \in P$, then we see that $\hat{\mu}$ takes root of unity values on $p$-singular elements. Conversely, suppose that $\hat{\mu}$ takes root of unity values on $p$-singular elements. If $1 \neq x \in P$ and $C=\mathbf{C}_{G}(x)$, then $\hat{\mu}(x)=\epsilon \mu^{*}(1)$ is a root of unity, and then $\mu^{*}(1)=1$. This proves the claim.

Next we prove that $\chi$ is primitive. Suppose that $\tau^{G}=\chi$, where $\tau \in \operatorname{Irr}(H)$. Since $\chi$ has $p^{\prime}$-degree, then $H$ contains a Sylow $p$-subgroup of $G$, which we may assume is $P$. We claim that $H$ is strongly $p$-embedded in $G$. Let $x \in H$ be a $p$-element. Since $G$ has a normal $p$-complement and abelian Sylow $p$-subgroups, by elementary group theory we know that $x^{g} \in H$ for some $g \in G$ if and only if $x^{g}=x^{h}$ for some $h \in H$. Then

$$
\chi(x)=(1 /|H|) \sum_{g \in G, x^{g} \in H} \tau\left(x^{g}\right)=(1 /|H|) m(x) \tau(x),
$$

where $m(x)=\left|\left\{g \in G \mid x^{g} \in H\right\}\right|$. Now, $x^{g} \in H$ if and only if $x^{g h} \in H$ for every $h \in H$, and we see that $m(x) /|H|$ is an integer dividing a root of unity. Then $m(x)=|H|$ by Lemma 2.1. Hence if $x^{g} \in H$, then $g \in H$, and therefore $H$ is strongly embedded in $G$, as claimed. In particular, if $1 \neq u \in P$ and $z \in \mathbf{C}_{G}(u)$, then $u \in H \cap H^{z}$ and therefore $z \in H$. Now, since $P \cong C_{p} \times C_{p}$, we may apply Theorem 6.2 .4 of [G], for example, to conclude that

$$
N \subseteq\left\langle\mathbf{C}_{G}(u) \mid 1 \neq u \in P\right\rangle \subseteq H
$$

and we deduce that $H=G$. This proves our claim that $\chi$ is primitive.

Now, suppose that $U$ is a proper normal subgroup of $N$ that admits $P$ and let $\theta \in \operatorname{Irr}(U)$ be $P$-invariant under $\mu$. Let $1 \neq x \in P$, and let $\mu^{*} \in \operatorname{Irr}\left(\mathbf{C}_{N}(x)\right)$ be the $\langle x\rangle$-Glauberman correspondent of $\mu$. By Theorem 13.29 of [I] , we have that $\theta^{*}$ lies under $\mu^{*}$, and we conclude that $\theta^{*}$ is also linear. Hence $\hat{\theta} \in \operatorname{Irr}(U P)$ also takes root of unity values on the $p$-singular elements of $U P$, and by induction we have that $\theta$ is linear.

Now, let $N / Z$ be a chief factor of $G$, and let $\theta \in \operatorname{Irr}(Z)$ be $P$-invariant under $\mu$, which we know is linear. Since $\hat{\mu}$ is primitive, then $\mu_{Z}$ is a multiple of $\theta$, so working in $G / \operatorname{ker}(\theta)$, we may assume that $Z \subseteq \mathbf{Z}(G)$ for every chief factor $N / Z$ of $G$. Suppose that $N / Z$ is abelian, and let $1 \neq x \in P$. Then $E / Z=\mathbf{C}_{N / Z}(x) \triangleleft G / Z$ (using that $P$ is abelian), and we deduce that $E=Z$ or $E=N$. If $E=N$, then $[N, x]=1$ by coprime action and $x \in \mathbf{O}_{p}(G)=1$. Therefore $P$ acts Frobeniusly on $N / Z$, but this is not possible because $P$ is not cyclic. So we may assume that $N$ is perfect. Suppose that $N / Z$ is a simple group. Since $\mathbf{O}_{p}(G / Z)=1$, it follows that $P$ is a subgroup of $\operatorname{Aut}(N / Z)$, which we know by the Classification of Finite Simple Groups has cyclic Sylow $p$-subgroups. This is not possible. Hence,

$$
N / Z=S_{1} / Z \times \cdots \times S_{k} / Z
$$

is a direct product of non-abelian simple groups which are permuted by $P$, where $k>1$, each $S_{i}$ is perfect and $\left[S_{i}, S_{j}\right]=1$. Write $S_{1}=S$. Now, since $N \subseteq \mathbf{N}_{G}(S)<$ $G$, let $1 \neq x \in P$ be such that $S^{x} \neq S$. Notice that $\langle x\rangle$ transitively permutes the elements of the set $\left\{S_{1} / Z, \ldots, S_{k} / Z\right\}$, because $S / Z<\prod_{y \in\langle x\rangle}\left(S_{i} / Z\right)^{y}$ is normal in $G$. Now define $\varphi: S \rightarrow \mathbf{C}_{N}(x)$ by

$$
\varphi(s)=s s^{x} \cdots s^{x^{p-1}} .
$$


This is a well-defined homomorphism. If $s \in \operatorname{ker}(\varphi)$, then $s \in Z$ (using that $G / Z$ is a direct product) and $s^{p}=1$, so $s=1$. Also, if $y \in \mathbf{C}_{N}(x)$, then, using again that $G / Z$ is a direct product, we have that $y=s s^{x} \cdots s^{x^{p-1}} z$ for some $s \in S$ and $z \in Z$. Now $z=z_{1}^{p}$ for some $z_{1} \in Z$ and $\varphi\left(s z_{1}\right)=y$. We conclude that $S \cong \mathbf{C}_{N}(x)$, and in particular that $\mathbf{C}_{N}(x)$ is perfect. Since $\mu^{*} \in \operatorname{Irr}\left(\mathbf{C}_{N}(x)\right)$ is linear, then $\mu^{*}=1$ and $\mu=1$ by the uniqueness of the Glauberman correspondence. Hence $\hat{\mu}$ is linear, as desired.

The following is Theorem A in the introduction.

Theorem 2.7. Let $G$ be a finite p-solvable group. Suppose that $S \in \operatorname{Syl}_{p}(G)$ has a non-cyclic subgroup of order $p^{2}$. Suppose that $\chi \in \operatorname{Irr}(G)$ is a faithful character that takes root of unity values on the p-singular elements of $G$. If $\mathbf{O}_{p}(G)=1$, then $\chi$ is linear.

Proof. Write $M=\mathbf{O}_{p^{\prime} p}(G)=N P$, where $N=\mathbf{O}_{p^{\prime}}(G)$ and $P \in \operatorname{Syl}_{p}(M)$. Since $p$ divides $|G|$ and $G$ is $p$-solvable, we have that $P>1$. By Hall-Higman's 1.2.3 Lemma, we have that $\mathbf{C}_{G}(N) \subseteq N$. We claim that $P$ is not cyclic. Otherwise, again by Hall-Higman's 1.2.3 Lemma, we would have that $\mathbf{C}_{G / N}(M / N) \subseteq M / N$. Since $\operatorname{Aut}(P)$ is abelian, then this implies that $G / M$ is abelian. But in this case, $G / M$ is a $p^{\prime}$-group and $P \in \operatorname{Syl}_{p}(G)$. This is not possible, since $S$ contains a non-cyclic subgroup of order $p^{2}$.

Now, by Lemma 2.3(b), we have that

$$
\chi_{M}=\sum_{i=1}^{s} \chi_{i}
$$

is a sum of different irreducible characters $\chi_{i} \in \operatorname{Irr}(M)$. Since $\chi$ has $p^{\prime}$-degree, it follows that $\left(\chi_{i}\right)_{N} \in \operatorname{Irr}(N)$ for all $i$. Let us write

$$
\chi_{N}=h \sum_{j=1}^{r} \mu_{j},
$$

where $\mu_{j} \in \operatorname{Irr}(N)$ are different. Notice that $s=h r$. By Lemma 2.3(c) we obtain that

$$
h r=s=\left[\chi_{M}, \chi_{M}\right]=1+\frac{h^{2} r-1}{|P|} .
$$

Hence

$$
(h r-1)|P|=\left(h^{2} r-1\right)=h(h r-1)+(h-1)
$$

and therefore $h r-1$ divides $h-1$. Then $h r-1 \leq h-1$, and we conclude that $r=1$. So $r=1, s=h$, and we have that $\chi_{N}=h \mu$ for some $\mu \in \operatorname{Irr}(N)$. Since $(h-1)|P|=h^{2}-1$, then $h=1$ or $h=|P|-1$.

If $h=1$, then we have that $\chi_{N}=\mu \in \operatorname{Irr}(N)$. In this case, let $V$ be a non-cyclic subgroup of $S$ of order $p^{2}$. Then $\chi_{N V} \in \operatorname{Irr}(N V)$, and we apply Theorem 2.6 to conclude that $\chi(1)=1$. Hence, we may assume that $h=|P|-1$.

Now, let $\hat{\mu} \in \operatorname{Irr}(M)$ be the canonical extension of $\mu$ to $M$, so that $\hat{\mu}$ is $G$-invariant (by using the uniqueness of the canonical extension). We have that $\chi_{i}=\hat{\mu} \lambda_{i}$ for some unique $\lambda_{i} \in \operatorname{Irr}(M / N)$, where the $\lambda_{i}$ are distinct. By Clifford's theorem and using that $G=M \mathbf{N}_{G}(P)$, we see that $\mathbf{N}_{G}(P)$ acts transitively on $\left\{\lambda_{1}, \ldots, \lambda_{s}\right\}$. Since $s=h=|P|-1$ we conclude that $\operatorname{Irr}(P)-\left\{1_{P}\right\}=\left\{\lambda_{1}, \ldots, \lambda_{s}\right\}$. In particular, 
$P$ is elementary abelian. We know that $P$ cannot be cyclic, so $P$ contains at least a non-cyclic subgroup of order $p^{2}$. Also

$$
\chi_{M}=\hat{\mu}\left(\sum_{\lambda \in \operatorname{Irr}(P)-\left\{1_{P}\right\}} \lambda\right) .
$$

Hence, if $z$ is a $p$-singular element of $M$, then $\chi(z)=-\hat{\mu}(z)$ and we have that $\hat{\mu}$ takes root of unity values on $p$-singular elements. Now, by Theorem 2.6 we have that $\mu$ is linear. Since $\chi$ is faithful and $\chi_{N}=\chi(1) \mu$, we conclude that $\mu$ is faithful, so $N \subseteq \mathbf{Z}(G)$. Since $\mathbf{C}_{G}(N) \subseteq N$, we conclude that $G$ is a $p^{\prime}$-group, and this is a final contradiction.

We mentioned in the introduction that our present Theorem A implies the module-theoretic result in $\mathrm{NR}$. In fact, if $V$ is a simple endo-trivial $K G$-module of a finite $p$-solvable group $G$ with $\mathbf{O}_{p}(G)=1, R$ is the usual complete local ring with $R / \mathbf{J}(R)=K$, then by using the Fong-Swan theorem, it is possible to lift $V$ to an $R G$-module $U$ that affords an irreducible $\chi \in \operatorname{Irr}(G)$. Now, by Lemma 1 of [T], it easily follows that $U \otimes U^{*} \cong 1 \oplus P$, where $P$ is a projective $R G$-module. Then $\chi(g) \bar{\chi}(g)-1=0$ for every $p$-singular $g \in G$ (using IV.2.5 in [F]), and we can apply Theorem A to $\chi$.

\section{REFERENCES}

[CMT] J. F. Carlson, N. Mazza and J. Thévenaz, Endotrivial modules for $p$-solvable groups, Trans. Amer. Math. Soc. 363 (2011), 4979-4996. MR2806698

[F] W. Feit, The Representation Theory of Finite Groups, North-Holland, 1982. MR661045 (83g:20001)

[G] D. Gorenstein, Finite Groups, Second edition, Chelsea Publishing Co., New York, 1980. MR569209 (81b:20002)

[GLS] D. Gorenstein, R. Lyons and R. Solomon, The Classification of the Finite Simple Groups, Number 3, American Mathematical Society Mathematical Surveys and Monographs, Volume 40, Number 3, AMS, Providence, RI, 1998. MR.1490581(98j:20011)

[I] M. Isaacs, Character Theory of Finite Groups, AMS Chelsea Publishing, Providence, RI, 2006. MR2270898

[N] G. Navarro, Characters and Blocks of Finite Groups, Cambridge University Press, 1998. MR.1632299 (2000a:20018)

[NR] G. Navarro and G. R. Robinson, On endo-trivial modules for $p$-solvable groups, Math. Z., doi:10.1007/s00209-010-0835-x, in press.

[R1] G. R. Robinson, A bound on norms of generalized characters with applications, J. Algebra 212 (1999), 660-668. MR1676858 (99m:20008)

[R2] G. R. Robinson, Endotrivial irreducible lattices, J. Algebra 335 (2011), 319-327. MR.2792579

[R3] G. R. Robinson, Generalized characters whose values on non-identity elements are roots of unity, Journal of Algebra 333 (2011), 458-464. MR2785954

[T] J. G. Thompson, Vertices and sources, J. Algebra 6 (1967), 1-6. MR0207863 (34:7677)

Departament D’Àlgebra, Universitat de València, 46100 Burjassot, Spain

E-mail address: gabriel.navarro@uv.es

Institute of Mathematics, University of Aberdeen, Aberdeen, AB24 3UE Scotland

E-mail address: g.r.robinson@abdn.ac.uk 